\title{
$\infty \sqrt{1}$ Stroke and \\ Cerebral venous sinus thrombosis after ChAdOx1 nCov-19 vaccination with a misleading first cerebral MRI scan
}

\author{
Benno Ikenberg (1) , ${ }^{1}$ Antonia Franziska Demleitner (1) ,, Thomas Thiele, ${ }^{2}$ \\ Benedikt Wiestler, ${ }^{3}$ Katharina Götze, ${ }^{4}$ Georg Mößmer, ${ }^{5}$ Paul Lingor (D) ${ }^{1}$
}

To cite: Ikenberg B, Demleitner AF, Thiele T, et al. Cerebral venous sinus thrombosis after ChAd0x1 nCov-19 vaccination with a misleading first cerebral MRI scan. Stroke \& Vascular Neurology 2021;0. doi:10.1136/ svn-2021-001095

$\mathrm{BI}$ and $\mathrm{AFD}$ contributed equally.

Received 3 May 2021

Accepted 9 June 2021
Check for updates

(c) Author(s) (or their employer(s)) 2021. Re-use permitted under CC BY-NC. No commercial re-use. See rights and permissions. Published by BMJ.

${ }^{1}$ Department of Neurology, Klinikum rechts der Isar der Technischen Universität München, Munchen, Germany ${ }^{2}$ Institut für Immunologie und Transfusionsmedizin, Universitätsmedizin Greifswald, Greifswald, Germany

${ }^{3}$ Department of Diagnostic und Interventional Neuroradiology, Klinikum rechts der Isar der Technischen Universität München, Munchen, Germany ${ }^{4}$ Department of Medicine III, Klinikum rechts der Isar der Technischen Universität München, Munchen, Germany ${ }^{5}$ Institute for Clinical Chemistry and Pathobiochemistry, Klinikum rechts der Isar der Technischen Universität München, Munchen, Germany

Correspondence to Dr Benno lkenberg; benno.ikenberg@tum.de

\section{SUMMARY}

Vaccine-induced immune thrombotic thrombocytopenia (VITT) and cerebral venous sinus thrombosis (CVST) have been recently described as rare complications following vaccination against SARS-CoV-2 with vector vaccines. We report a case of a young woman who presented with VITT and cerebral CVST 7 days following vaccination with ChAd0x1 nCov-19 (AstraZeneca). While the initial MRI was considered void of pathological findings, MRI 3 days later revealed extensive CVST of the transversal and sigmoidal sinus with intracerebral haemorrhage. Diagnostic tests including a platelet-factor-4-induced platelet activation assay confirmed the diagnosis of VITT. Treatment with intravenous immunoglobulins and argatroban resulted in a normalisation of platelet counts and remission of CVST.

\section{BACKGROUND}

Active vaccination against SARS-CoV-2 is currently one of the most important measures to contain the COVID-19 pandemic. With increasing numbers of ChAdOx1 nCov-19 (AstraZeneca) vaccinations, adverse events such as thrombotic thrombocytopenia and cerebral venous sinus thrombosis (CVST) were observed as very rare complications. ${ }^{1}$ Vaccine-induced thrombotic thrombocytopenia (VITT) mediated by platelet-activating antibodies against platelet factor 4 (PF4) was observed and considered causative for such vascular events. ${ }^{2}$ Here, we report a case of VITT causing a CVST in an otherwise healthy young woman following ChAdOx1 nCov-19 vaccination and highlight particular laboratory and imaging features which may advise clinical decision making in the next weeks.

\section{CASE PRESENTATION}

A young woman in the early 30 s presented to our emergency department with an isolated headache (Numerical Rating Scale (NRS) score $=5)$. The patient denied any premedication (including oral contraception or prior heparin treatment), vascular risk factors, and pre-existing disease or family history of any thrombotic event. She had the first dose of ChAdOx1 nCov-19 vaccination 7 days before and reported mild myalgia, holocephalic headache and chills, which subsided within 24 hours. The neurological examination was normal. Particularly, no meningism and no petechia were observed. Laboratory results showed an otherwise unexplained thrombocytopenia of $97000 / \mu \mathrm{L}$ and a $\mathrm{C}$ reactive protein of $8.1 \mathrm{mg} / \mathrm{dL}$ (figure $1 \mathrm{~A}$ ). A cerebral MRI scan was without pathological finding, in particular without any clear sign for CVST (figure 1B-1). The patient was discharged from our hospital on the same day.

\section{INVESTIGATIONS}

Three days later, the patient was referred to our department with persisting headaches (NRS score $=8$ ), progressive thrombocytopenia of $37000 / \mu \mathrm{L}$ and an increased D-dimer concentration of $12859 \mu \mathrm{g} / \mathrm{L}$ fibrinogen equivalent unit (FEU) (reference range: $<500 \mu \mathrm{g} / \mathrm{L}$ FEU). The screening test for heparin-induced thrombocytopenia (HIT) was positive (particle gel agglutination immunoassay for antibodies against PF4/heparin, polyvalent, ie, not specific for IgG antibodies; ID-PaGIA Heparin/PF4 Antibody Test, DiaMed). The PCR test for SARS-CoV-2 from a nasopharyngeal swab was negative. On clinical examination, the patient presented with a discrete gait ataxia and reported progressive amnestic difficulties as well as discrete amnesic aphasia. A new MRI scan revealed CVST of the left transverse and sigmoidal sinus with a left-temporal and left-cerebellar intracerebral haemorrhage (figure 1B-2). The results of the heparin-induced platelet activation assay (HIPA) and the plateletfactor-4-induced platelet activation assay (PIPA, a modified HIPA test) showed strong IgG-receptor-mediated platelet activation in both the presence and absence of heparin confirming the diagnosis of VITT. In addition, a hypercoagulable state work-up showed no relevant findings. 
A

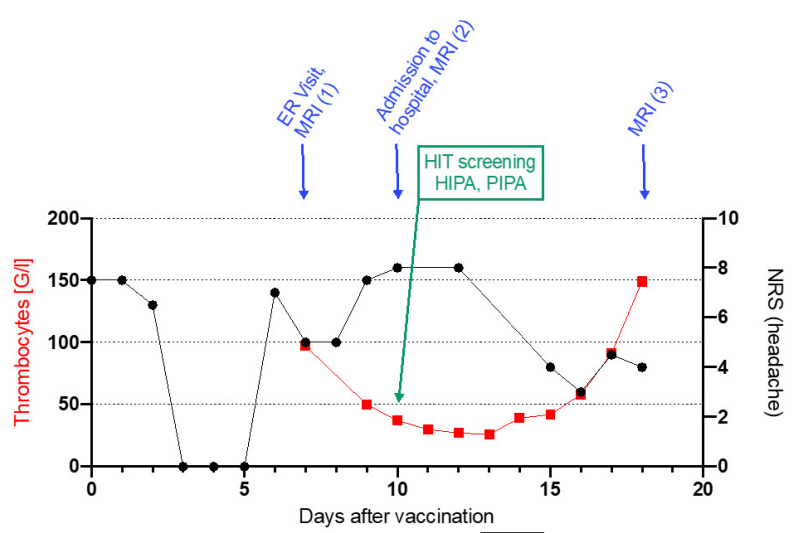

IVIG

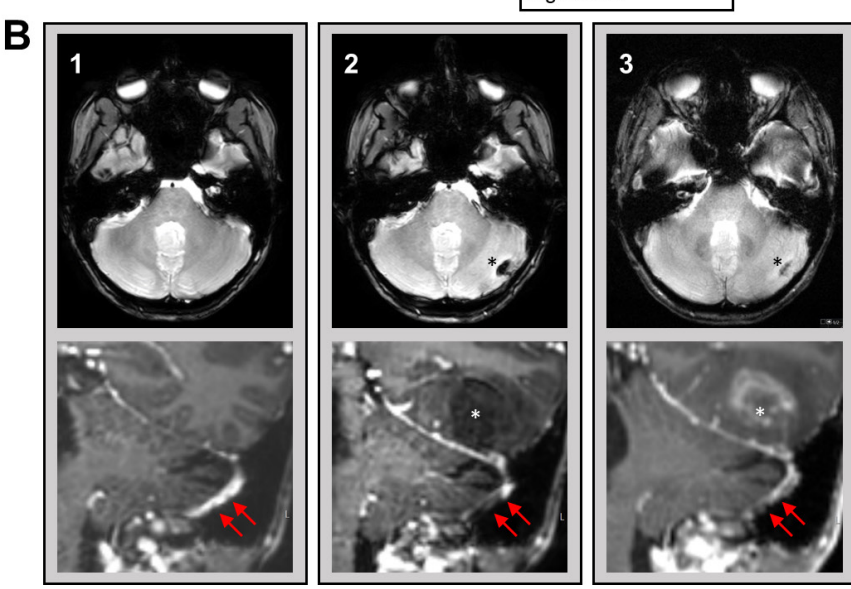

Figure 1 A time course of clinical characteristics and laboratory results following vaccination. Platelet count is depicted in red. The value of day 9 prior to second presentation in our hospital was provided by the generalist. Headache intensity is shown in black according to the Numerical Rating Scale. (B) Serial cerebral MRI scans. Upper row: axial T2* sequence of the infratentorial brain; lower row: coronal T1w sequence of the cerebellum, temporal lobe and sinus transversus. (B-1) MRI scan at first presentation in our emergency department with thrombocytopenia and headache. At first look, the MRI scan was without a clear sign for CVST. Retrospectively (red arrows), a slight irregularity as indicator of beginning thrombosis may be discussed. (B-2) First follow-up MRI 3 days later revealed a CVST of the left transverse and sigmoidal sinus (red arrows) with a left-temporal and left cerebellar intracerebral haemorrhage (asterisks). (B-3) Most recent follow-up MRI showing a persisting CVST and intracerebral haemorrhage with a slightly progressive perifocal oedema. Volume of cerebellar haemorrhage slightly decreased. CVST, cerebral venous sinus thrombosis; ER, emergency room; HIPA, heparininduced platelet activation assay; HIT, heparin-induced thrombocytopenia; IVIG, intravenous immunoglobulin; PIPA, platelet-factor-4-induced platelet activation assay.

\section{TREATMENT}

The patient was admitted to our stroke unit; anticoagulation with argatroban (Argatra) was initiated immediately; and intravenous immunoglobulin therapy $(1 \mathrm{~g} / \mathrm{kg}$ body weight/day for 2 days) was applied for treatment of suspected VITT.

\section{OUTCOME AND FOLLOW-UP}

Under continuous anticoagulation with argatroban, the patient improved with regressive headache but persistent minimal gait ataxia, amnestic deficits as well as discrete amnesic aphasia. A follow-up MRI scan showed persisting CVST but regressive cerebellar haemorrhage with a slightly progressive temporal perifocal oedema (figure 1B-3). Subsequently, the platelet count increased in the following days (figure 1A).

\section{DISCUSSION}

Consistent with the initial description of VITT following ChAdOx1 nCov-19 vaccination, ${ }^{12}$ we report on a young woman with unremarkable medical history suffering from CVST. The delay between vaccination and symptom onset was 7 days, which is in the range of 5-16 days reported for VITT. A positive PIPA confirmed the diagnosis.

Following positive HIT screening test, treatment of CVST was started with argatroban. Because of continuous clinical improvement, this treatment was continued before switching the patient to anticoagulation with dabigatran (Pradaxa) on day 8 after hospitalisation. According to current recommendations, ${ }^{3}$ we treated the patient with intravenous immunoglobulins for 2 days. Subsequently, the platelet count increased continuously.

Although our patient suffered from headache and mild thrombocytopenia at first presentation, the initial MRI scan was without any clear pathological finding. The follow-up MRI scan, however, revealed an extensive CVST. There is an ongoing debate about the appropriate imaging modality for detecting CVST. In a recent meta-analysis, ${ }^{4}$ CT and MRI had similar diagnostic performance. Retrospectively, we noted small irregularities at the bottom of the left transverse sinus in the contrast-enhanced T1w image (figure 1B-1). In view of the clinical course, this warrants a high sensitivity also for (small) abnormalities in cerebral veins and sinuses in these patients. The clinical challenge of managing such patients and the importance of high vigilance are also supported by a similar patient who also deteriorated at second presentation to the hospital in a recent case series. ${ }^{1}$

Given the high number of patients presenting to emergency departments with headache or other unspecific symptoms following vaccination with ChAdOx1 nCov-19 or other vector vaccines for SARS-CoV-2, our case advocates to give high priority to patients with signs of thrombocytopenia and/or elevated D-dimer. Importantly, our case demonstrates that an initial MRI scan can be normal and a re-examination should be performed if clinical suspicion persists. Progression of thrombocytopenia should be monitored, while a platelet substitution is not recommended as it could further aggravate thrombosis and thrombocytopenia. ${ }^{3}$ Although our HIT test was positive, it is important to note that commercially available tests are not sufficient to exclude/confirm VITT so far and literature should be screened regularly for availability of new tests/recommendations ${ }^{3}$ in this rapidly evolving field. Given the high number of vaccinations 
with ChAdOx1 nCov-19, the occurrence of VITT and subsequent CVST has to be considered a very rare complication: according to a recent European Medicines Agency report as of 4 April 2021, 34 million people have been vaccinated with ChAdOx1 nCov-19 (Vaxzevria, formerly COVID-19 Vaccine, AstraZeneca), and 169 cases of CVST and 53 cases of splanchnic vein thrombosis were recorded by EudraVigilance. ${ }^{5}$ Our case report intends to raise alertness in emergency departments and to provide a diagnostic and therapeutic work-up for this rare complication of vector vaccines against SARS-CoV-2.

\section{Learning points}

- High priority should be given to patients presenting with headaches following ChAd0x1 nCov-19 vaccination.

- Screening for thrombocytopenia and/or elevated D-dimer is mandatory in such patients.

- Initial cerebral MRI scans can be normal-if clinical suspicion for sinus vein thrombosis persists, a repeated MRI scan should be performed in patients following ChAd0x1 nCov-19 vaccination.

- Current commercially available heparin-induced thrombocytopenia screening tests are not sufficient to diagnose vaccine-induced thrombotic thrombocytopenia.

Acknowledgements We are indebted to the patient who gave informed consent for publication of this case report.

Contributors Treating neurologists: BI, AFD and PL; MRI analysis and interpretation: BW; laboratory tests and interpretation: TT, KG and GM; first draft: $\mathrm{Bl}$; supervision: PL. All authors revised and approved the final version of the manuscript.
Funding The authors have not declared a specific grant for this research from any funding agency in the public, commercial or not-for-profit sectors.

Competing interests None declared.

Patient consent for publication Not required.

Provenance and peer review Not commissioned; internally peer reviewed.

Open access This is an open access article distributed in accordance with the Creative Commons Attribution Non Commercial (CC BY-NC 4.0) license, which permits others to distribute, remix, adapt, build upon this work non-commercially, and license their derivative works on different terms, provided the original work is properly cited, appropriate credit is given, any changes made indicated, and the use is non-commercial. See: http://creativecommons.org/licenses/by-nc/4.0/.

\section{ORCID iDs}

Benno Ikenberg http://orcid.org/0000-0002-0798-7149

Antonia Franziska Demleitner http://orcid.org/0000-0001-9557-9243

Paul Lingor http://orcid.org/0000-0001-9362-7096

\section{REFERENCES}

1 Schultz NH, Sørvoll IH, Michelsen AE, et al. Thrombosis and thrombocytopenia after ChAdOx1 $\mathrm{nCoV}-19$ vaccination. $N$ Engl J Med 2021;384:2124-30.

2 Greinacher A, Thiele T, Warkentin TE, et al. Thrombotic thrombocytopenia after ChAdOx1 nCov-19 vaccination. N Engl J Med 2021;384:2092-101.

3 Oldenburg J, Klamroth R, Langer F. Diagnosis and management of vaccine-related thrombosis following AstraZeneca COVID-19 vaccination: guidance statement from the GTH. Hamostaseologie 2021.

4 Xu W, Gao L, Li T, et al. The performance of CT versus MRI in the differential diagnosis of cerebral venous thrombosis. Thromb Haemost 2018;118:1067-77.

5 AstraZeneca's COVID-19 vaccine: EMA finds possible link to very rare cases of unusual blood clots with low blood platelets. Available: https://www.ema.europa.eu/en/news/astrazenecas-covid-19-vaccineema-finds-possible-link-very-rare-cases-unusual-blood-clots-lowblood [Accessed 16 Apr 2021]. 